\title{
Low-Rank Solution of Convex Relaxation for Optimal Power Flow Problem
}

\author{
Somayeh Sojoudi*, Ramtin Madani ${ }^{+}$and Javad Lavaei ${ }^{+}$ \\ *Department of Computing and Mathematical Sciences, California Institute of Technology \\ ${ }^{+}$Department of Electrical Engineering, Columbia University
}

\begin{abstract}
This paper is concerned with solving the nonconvex problem of optimal power flow (OPF) via a convex relaxation based on semidefinite programming (SDP). We have recently shown that the SDP relaxation has a rank-1 solution from which the global solution of OPF can be found, provided the power network has no cycle. The present paper aims to provide a better understating of the SDP relaxation for cyclic networks. To this end, an upper bound is derived on rank of the minimumrank solution of the SDP relaxation, which depends only on the topology of the power network. This bound is expected to be very small in practice due to the mostly planar structure of realworld networks. A heuristic method is then proposed to enforce the low-rank solution of the SDP relaxation to become rank-1. To elucidate the efficacy of this technique, it is proved that this method works for weakly-cyclic networks with cycles of size 3 . Although this paper mainly focuses on OPF, the results developed here can be applied to several OPF-based emerging optimizations for future electrical grids.
\end{abstract}

\section{INTRODUCTION}

The real-time operation of a power network depends heavily on various large-scale optimization problems solved from every few minutes to every several months. State estimation, optimal power flow (OPF), security-constrained OPF, and transmission planning are some fundamental operations solved for transmission networks. Although most of the energyrelated optimizations are traditionally solved at transmission level, there are a few optimizations associated with distribution systems, e.g., sizing of capacitor banks and network reconfiguration. Each of these problems has the power flow equations embedded in it. With the exception of the security-constrained OPF, these problems often have less than 10,000 variables. Even though the number of variables in these optimizations is modest compared to many real-world optimizations involving millions of variables, it is very challenging to solve energyrelated optimization problems efficiently. This is in part due to the nonlinearities imposed by the laws of physics.

The OPF problem is the most fundamental optimization for power systems, which aims to find an optimal operating point of the power network minimizing a certain objective function (e.g., power loss or generation cost) subject to network and physical constraints [1]. Due to the nonlinear interrelation among active power, reactive power and voltage magnitude, $\mathrm{OPF}$ is described by nonlinear equations and may have a nonconvex feasibility region [2]. Since 1962, this problem has been studied thoroughly, and various heuristic and local-search algorithms have been developed [3], [4].

Emails: sojoudi@caltech.edu; rm3122@columbia.edu; lavaei@ee.columbia.edu.
The paper [5] proposes two methods to solve an OPF problem: (i) using a convex relaxation based on semidefinite programming (SDP), and (ii) solving the SDP-type Lagrangian dual of OPF. That work shows that the SDP relaxation is exact if and only if the duality gap is zero. More importantly, [5] makes the observation that OPF has a zero duality gap for IEEE benchmark systems with 14, 30, 57, 118 and 300 buses, in addition to several randomly generated power networks. This technique is the first method proposed since the introduction of the OPF problem that is able to find a provably global solution for practical OPF problems. The convex relaxation proposed in [5] has been generalized to more complicated problems, such as security constrained OPF, in [6].

By exploiting the passivity of transmission lines and transformers, the paper [7] shows that the SDP relaxation is exact in two cases: (i) for acyclic networks, and (ii) for cyclic networks after relaxing the angle constraints via the inclusion of actual/virtual phase shifters (similar result was derived in [8] and [9] for acyclic networks). Although the SDP relaxation works for the above-mentioned IEEE cyclic systems, it may not be exact for every mesh network (see the three-bus example contrived in [10]). The present paper aims to explore the limitations of the SDP relaxation for mesh networks.

The exactness of the SDP relaxation amounts to the existence of a rank-1 solution for this problem. In the case when the SDP relaxation is not exact, its solution may still be useful if its rank is low compared to the size of the network. Since the SDP relaxation may not be exact for mesh networks, the first goal of this work is to study the rank of the minimum-rank solution of the SDP relaxation (under the load over-satisfaction assumption [5]). To this end, an upper bound is derived on this number, which depends only on the topology of the network. This number is expect to be very small in practice due to the mostly planar structure of real-world power networks. A question arises as to whether this low-rank solution can be converted to a rank-1 solution leading to a nearly global solution of OPF. To address this problem, a heuristic algorithm is proposed whose efficacy is demonstrated in simulations. Moreover, it is proved that this method is guaranteed to work for certain weakly-cyclic networks.

The SDP relaxation for OPF has attracted much attention due to its ability to find a global solution in polynomial time, and has been applied to various applications in power systems including: voltage regulation in distribution systems [11], state estimation [12], calculation of voltage stability margin [13], economic dispatch in unbalanced distribution networks [14], power management under time-varying conditions 
[15], distributed energy management [16], OPF with storage integration [17], and charging of electric vehicles [18]. The results of this work enhance the theoretical foundation of the convex relaxation for all of the above problems as well as emerging applications related to renewable energy, distributed generation, and demand response.

Notations: $\mathbb{R}, \mathbb{H}^{n}$, and $\mathbb{H}_{+}^{n}$ denote the sets of real numbers, $n \times n$ Hermitian matrices, and $n \times n$ positive semidefinite Hermitian matrices, respectively. $\operatorname{Re}\{\mathbf{W}\}, \operatorname{Im}\{\mathbf{W}\}, \operatorname{rank}\{\mathbf{W}\}$, and trace $\{\mathbf{W}\}$ denote the real part, imaginary part, rank, and trace of a given scalar/matrix $\mathbf{W}$, respectively. The notation $\mathbf{W} \succeq 0$ means that $\mathbf{W}$ is Hermitian and positive semidefinite. The notation " $\mathrm{i}$ " is reserved for the imaginary unit. The notation $\measuredangle x$ denotes the angle of a complex number $x$. The symbol “*” represents the conjugate transpose operator. Given a matrix $\mathbf{W}$, its $(l, m)$ entry is denoted as $W_{l m}$. The superscript $(\cdot)^{\text {opt }}$ is used to show the optimal value of an optimization parameter.

\section{Optimal POWER Flow}

Consider a power network with the set of buses $\mathcal{N}:=$ $\{1,2, \ldots, n\}$, the set of generator buses $\mathcal{O} \subseteq \mathcal{N}$ and the set of flow lines $\mathcal{L} \subseteq \mathcal{N} \times \mathcal{N}$, where:

- A known constant-power load with the complex value $P_{D_{k}}+Q_{D_{k}} \mathrm{i}$ is connected to each bus $k \in \mathcal{N}$.

- A generator with an unknown complex output $P_{G_{k}}+$ $Q_{G_{k}} \mathrm{i}$ is connected to each bus $k \in \mathcal{O}$.

- Each line $(l, m) \in \mathcal{L}$ of the network is modeled as a passive device with an admittance $y_{l m}$ (the network can be modeled as a general admittance matrix).

The goal is to design the unknown outputs of all generators in such a way that the load constraints are satisfied. To formulate this problem, named optimal power flow $(O P F)$, define:

- $V_{k}$ : Unknown complex voltage at bus $k \in \mathcal{N}$.

- $P_{l m}$ : Unknown active power transferred from bus $l \in \mathcal{N}$ to the rest of the network through the line $(l, m) \in \mathcal{L}$.

- $S_{l m}$ : Unknown complex power transferred from bus $l \in$ $\mathcal{N}$ to the rest of the network through the line $(l, m) \in \mathcal{L}$.

- $f_{k}\left(P_{G_{k}}\right)$ : Known convex function representing the generation cost for generator $k \in \mathcal{O}$.

Define $\mathbf{V}, \mathbf{P}_{G}, \mathbf{Q}_{G}, \mathbf{P}_{D}$ and $\mathbf{Q}_{D}$ as the vectors $\left\{V_{k}\right\}_{k \in \mathcal{N}}$, $\left\{P_{G_{k}}\right\}_{k \in \mathcal{O}},\left\{Q_{G_{k}}\right\}_{k \in \mathcal{O}},\left\{P_{D_{k}}\right\}_{k \in \mathcal{N}}$ and $\left\{Q_{D_{k}}\right\}_{k \in \mathcal{N}}$, respectively. Given the known vectors $\mathbf{P}_{D}$ and $\mathbf{Q}_{D}$, OPF minimizes the total generation cost $\sum_{k \in \mathcal{O}} f_{k}\left(P_{G_{k}}\right)$ over the unknown parameters $\mathbf{V}, \mathbf{P}_{G}$ and $\mathbf{Q}_{G}$ subject to the power balance equations at all buses and some network constraints. To simplify the formulation of OPF, with no loss of generality assume that $\mathcal{O}=\mathcal{N}$. The mathematical formulation of OPF is given in (1), where:

- (1a) and (1b) are the power balance equations accounting for the conservation of energy at bus $k$.

- (1c), (1d) and (1e) restrict the active power, reactive power and voltage magnitude at bus $k$, for the given limits $P_{k}^{\min }, P_{k}^{\max }, Q_{k}^{\min }, Q_{k}^{\max }, V_{k}^{\min }, V_{k}^{\max }$.

- Each line of the network is subject to one of the four capacity constraints given in (3), for given upper bounds $\theta_{l m}^{\max }=\theta_{m l}^{\max }, P_{l m}^{\max }=P_{m l}^{\max }, S_{l m}^{\max }=S_{m l}^{\max }$ and $\Delta V_{l m}^{\max }=\Delta V_{m l}^{\max }$ (we consider $\theta_{l m}^{\max }$ less than $90^{\circ}$ due to the current practice in power networks).

- $\mathcal{N}(k)$ denotes the set of the neighboring nodes of bus $k$.

\section{CONVEX Relaxation}

Consider the OPF problem (1) subject to one of the capacity constraints given in (3). This problem is nonconvex due to the nonlinear terms $\left|V_{k}\right|$ 's and $V_{k} V_{l}^{*}$ 's. Since this problem is NPhard in the worst case, the paper [5] suggests solving a convex relaxation of OPF. To this end, notice that the constraints of OPF can all be expressed as linear functions of the entries of the quadratic matrix $\mathbf{V V}^{*}$. This implies that if the matrix $\mathbf{V V}^{*}$ is replaced by a new matrix variable $\mathbf{W} \in \mathbb{H}^{n}$, then the constraints of OPF become convex in W. Since $\mathbf{W}$ plays the role of $\mathbf{V V}^{*}$, two constraints must be added to the reformulated OPF problem in order to preserve the equivalence of the two formulations: (i) $\mathbf{W} \succeq 0$, and (ii) $\operatorname{rank}\{\mathbf{W}\}=1$. Observe that Constraint (ii) is the only nonconvex constraint of the reformulated OPF problem. Inspired by this observation, the SDP relaxation of OPF is defined as the OPF problem reformulated in terms of $\mathbf{W}$ under the additional constraint $\mathbf{W} \succeq 0$. This problem is provided in (2) and (4). If the SDP relaxation has a rank-1 solution $\mathbf{W}^{\text {opt }}$, then it is said that the relaxation is exact. The exactness of the SDP relaxation is a desirable property being sought, because it makes the convex SDP relaxation and the nonconvex OPF problem equivalent.

\section{LOW-RANK SOLUTION}

Definition 1: Define $\eta$ as the minimum number of vertices whose removal from the power network eliminates all cycles of the network.

To illustrate the definition of $\eta$, observe that this number is equal to 0 if the network is acyclic and is equal to 1 if all cycles of the network share a common node.

Definition 2: A subgraph $\mathcal{S}=\left(\mathcal{N}_{S}, \mathcal{L}_{S}\right)$ of the power network is called an induced subgraph if $\mathcal{N}_{S} \subseteq \mathcal{N}$ and $\mathcal{L}_{S}=\left\{(l, m) \mid l, m \in \mathcal{N}_{S}\right.$ and $\left.(l, m) \in \mathcal{L}\right\}$. A maximum induced subtree (subjungle) of the network refers to an induced subgraph $\mathcal{S}$ that is a tree (jungle) with the maximum number of vertices.

According to the definition of $\eta$, a maximum induced subjungle of the power network has $n-\eta$ vertices.

Definition 3: Given a positive number $r$ and a matrix $\mathbf{A} \in$ $\mathbb{H}_{+}^{r}$, define $\mathcal{G}(\mathbf{A})$ as a graph with the vertex set $\mathcal{N}_{r}=\{1, \ldots, r\}$ and the edge set $\left\{(l, m) \mid A_{l m} \neq 0,(l, m) \in \mathcal{N}_{r} \times \mathcal{N}_{r}\right\}$.

Definition 4: Two simple graphs $\mathcal{G}_{1}$ and $\mathcal{G}_{2}$ are called equivalent if there exists an edge-preserving bijection between their vertex sets. The notation $\mathcal{G}_{1} \equiv \mathcal{G}_{2}$ shows this equivalence.

The following lemma has been borrowed from [19].

Lemma 1: Consider a graph $\mathcal{G}$ with a maximum induced subtree $\mathcal{T}$. Given a matrix $\mathbf{A} \in \mathbb{H}_{+}^{|\mathcal{G}|}$ with the property $\mathcal{G}(\mathbf{A}) \equiv$ $\mathcal{G}$, the relation $\operatorname{rank}\{\mathbf{A}\} \geq|\mathcal{T}|-1$ holds.

The power balance equations (1a) and (1b) are equality constraints. One may relax these equations to inequality constraints so that each bus $k \in \mathcal{N}$ can be oversupplied. This 


\begin{tabular}{|c|c|}
\hline OPF Problem & SDP Relaxation of OPF \\
\hline $\begin{array}{l}\text { Minimize } \sum_{k \in \mathcal{O}} f_{k}\left(P_{G_{k}}\right) \text { over } \mathbf{P}_{G}, \mathbf{Q}_{G}, \mathbf{V} \\
\text { Subject to: }\end{array}$ & $\begin{array}{l}\text { Minimize } \sum_{k \in \mathcal{O}} f_{k}\left(P_{G_{k}}\right) \text { over } \mathbf{P}_{G}, \mathbf{Q}_{G}, \mathbf{W} \in \mathbb{H}_{+}^{n} \\
\text { Subject to: }\end{array}$ \\
\hline 1- A capacity constraint for each line $(l, m) \in \mathcal{L}$ & 1- A convexified capacity constraint for each line \\
\hline 2- The following constraints for each bus $k \in \mathcal{N}$ : & 2- The following constraints for each bus $k \in \mathcal{N}$ : \\
\hline$P_{G_{k}}-P_{D_{k}}=\sum_{l \in \mathcal{N}(k)} \operatorname{Re}\left\{V_{k}\left(V_{k}^{*}-V_{l}^{*}\right) y_{k l}^{*}\right\}$ & $P_{G_{k}}-P_{D_{k}}=\sum_{l \in \mathcal{N}(k)} \operatorname{Re}\left\{\left(W_{k k}-W_{k l}\right) y_{k l}^{*}\right\}$ \\
\hline$Q_{G_{k}}-Q_{D_{k}}=\sum_{l \in \mathcal{N}(k)} \operatorname{Im}\left\{V_{k}\left(V_{k}^{*}-V_{l}^{*}\right) y_{k l}^{*}\right\}(1 \mathrm{~b})$ & $Q_{G_{k}}-Q_{D_{k}}=\sum_{l \in \mathcal{N}(k)} \operatorname{Im}\left\{\left(W_{k k}-W_{k l}\right) y_{k l}^{*}\right\}(2 \mathrm{~b})$ \\
\hline$P_{k}^{\min } \leq P_{G_{k}} \leq P_{k}^{\max }$ & $P_{k}^{\min } \leq P_{G_{k}} \leq P_{k}^{\max }$ \\
\hline$Q_{k}^{\min } \leq Q_{G_{k}} \leq Q_{k}^{\max }$ & $Q_{k}^{\min } \leq Q_{G_{k}} \leq Q_{k}^{\max }$ \\
\hline$V_{k}^{\min } \leq\left|V_{k}\right| \leq V_{k}^{\max }$ & $\left(V_{k}^{\min }\right)^{2} \leq W_{k k} \leq\left(V_{k}^{\max }\right)^{2}$ \\
\hline
\end{tabular}

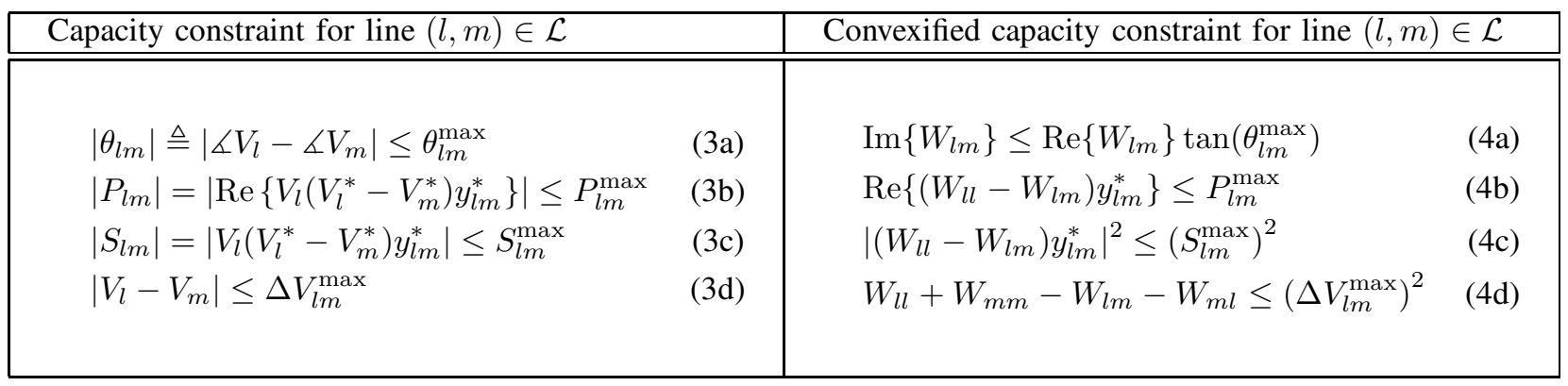

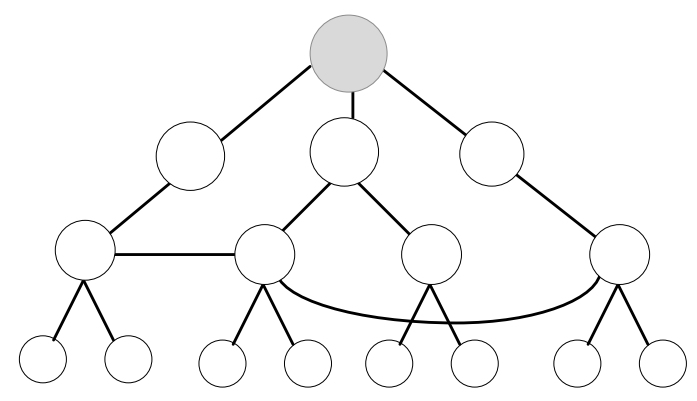

Fig. 1: A graph with $\eta=1$.

notion is called over-satisfaction and has been considered in a number of papers (see [5], [3] and the references therein). The main idea is that whenever a power network operates under a normal condition, it is expected that the solution of the OPF problem remains intact or changes insignificantly under the load over-satisfaction assumption.

In this part, we make the practical assumption that each power line in the network is a passive device with nonnegative resistance and inductance.

Theorem 1: Consider the OPF problem given in (1) subject to the capacity constraint (3a), (3b) or (3d) and under the load over-satisfaction assumption. If this problem is feasible, then its corresponding SDP relaxation has a solution $\left(\mathbf{W}^{\mathrm{opt}}, \mathbf{P}_{G}^{\mathrm{opt}}, \mathbf{Q}_{G}^{\mathrm{opt}}\right)$ such that

$$
\operatorname{rank}\left\{\mathbf{W}^{\mathrm{opt}}\right\} \leq \eta+1
$$

Sketch of proof: Let $\mathcal{J}$ denote a maximum induced subjungle of the power network. One can expand the subjungle $\mathcal{J}$ into a tree $\mathcal{T}$ by adding a minimal set of additional edges to this possibly disconnected subgraph. Notice that $|\mathcal{J}|=|\mathcal{T}|=n-\eta$. Denote the edge set of $\mathcal{T}$ as $\mathcal{L}_{T}$. Since the OPF problem is feasibly by assumption, there exists an optimal solution $\left(\mathbf{W}^{0}, \mathbf{P}_{G}^{\mathrm{opt}}, \mathbf{Q}_{G}^{\mathrm{opt}}\right)$ for this problem. Now, consider the optimization problem:

$$
\begin{aligned}
& \min _{\mathbf{W} \in \mathbb{H}_{+}^{n}}-\varepsilon \sum_{(l, m) \in \mathcal{L}_{T}} \operatorname{Re}\left\{W_{l m}\right\} \\
& \text { s.t. } W_{k k}=W_{k k}^{0}, \quad k \in \mathcal{N} \\
& \operatorname{Re}\left\{W_{l m}\right\} \geq \operatorname{Re}\left\{W_{l m}^{0}\right\}, \quad(l, m) \in \mathcal{L} \\
& \operatorname{Im}\left\{W_{l m}\right\}=\operatorname{Im}\left\{W_{l m}^{0}\right\}, \quad(l, m) \in \mathcal{L}
\end{aligned}
$$

where $\varepsilon$ is an arbitrary strictly positive number. Let $\mathbf{W}^{\text {opt }}$ denote an arbitrary solution of the above optimization. Since 
the resistance and inductance of each line $(l, m) \in \mathcal{L}$ are both nonnegative numbers, it is straightforward to verify that $\left(\mathbf{W}^{\mathrm{opt}}, \mathbf{P}_{G}^{\mathrm{opt}}, \mathbf{Q}_{G}^{\mathrm{opt}}\right)$ is an optimal solution of the SDP relaxation under the load over-satisfaction assumption. Now, it remains to prove that $\mathbf{W}^{\text {opt }}$ satisfies the inequality (5).

To proceed with the proof, we need to take the Lagrangian of Optimization (6). Let $\mathbf{A} \in \mathbb{H}_{+}^{n}$ denote the dual variable corresponding to the constraint $\mathbf{W} \succeq 0$. It can be shown that $\mathcal{G}(\mathbf{A}) \equiv \mathcal{T}$. Hence, it follows from Lemma 1 that

$$
\operatorname{rank}\left\{\mathbf{A}^{\mathrm{opt}}\right\} \geq|\mathcal{T}|-1=n-\eta-1 \text {. }
$$

On the other hand, the complementary slackness condition trace $\left\{\mathbf{W}^{\text {opt }} \mathbf{A}^{\text {opt }}\right\}=0$ yields that

$$
\operatorname{rank}\left\{\mathbf{A}^{\mathrm{opt}}\right\}+\operatorname{rank}\left\{\mathbf{W}^{\mathrm{opt}}\right\} \leq n .
$$

The proof is completed by combining (7) and (8).

Note that Theorem 1 does not apply to the capacity constraint (3c), and hence one of its almost equivalent counterparts (3a), (3b) or (3d) should be deployed in the SDP relaxation.

There is a large body of literature on computing $\eta$, which signifies that this number is small for a very broad class of graphs. To illustrate the application of Theorem 1, consider the distribution network depicted in Figure 1. This network has two cycles, which can be used to exchange renewable energy between the load buses without going through the feeder (the node shown in gray). Since removing the feeder node eliminates all cycles of the network, it follows from Theorem 1 that the SDP relaxation of OPF has a (hidden) solution $\left(\mathbf{W}^{\text {opt }}, \mathbf{P}_{G}^{\mathrm{opt}}, \mathbf{Q}_{G}^{\mathrm{opt}}\right)$ with the property $\operatorname{rank}\left\{\mathbf{W}^{\mathrm{opt}}\right\} \leq 2$. Having been aware of such a low-rank solution, the following question arises: how can the SDP relaxation be enforced to return a rank-1 solution? Motivated in part by the proof of Theorem 1, we propose a heuristic method below.

Perturbed SDP relaxation: This optimization is obtained from the SDP relaxation of the OPF problem by replacing its objective function with

$$
\sum_{k \in \mathcal{G}} f_{k}\left(P_{G_{k}}\right)-\varepsilon \sum_{(l, m) \in \mathcal{L}} \operatorname{Re}\left\{W_{l m}\right\}
$$

for a given positive number $\varepsilon$.

The perturbed SDP relaxation aims to increase the real part of some entries of $\mathbf{W}$ with the hope of making this matrix rank-1 at optimality. In particular, the larger the value of $\varepsilon$ is, the higher penalty is placed on the rank of $\mathbf{W}$. We have witnessed the efficacy of this technique in extensive simulations and will report its performance in two simulations later in this paper.

In what follows, we aim to prove that the above technique is guaranteed to work for networks with small cycles.

Definition 5: A graph is called weakly cyclic if every edge of the graph belongs to at most one cycle in the graph.

Theorem 2: Consider a weakly-cyclic lossless network with cycles of size 3 . Given an arbitrary strictly positive number $\varepsilon$, every solution of the perturbed SDP relaxation has rank-1, provided $Q_{k}^{\min }=-\infty$ for every $k \in \mathcal{N}$.

Proof: The proof has been omitted for brevity.

Theorem 2 can be similarly extended to lossy networks.

\section{NumERICAL EXAMPLES}

Example 1: Consider a ring network with 10 nodes and 10 links $(1,2),(2,3), \ldots,(9,10),(10,1)$. Suppose that the nodes $4,5,6,9$ and 10 are generator buses while the remaining nodes are load buses. Let the cost function $f_{k}\left(P_{G_{k}}\right)$ be chosen as $c_{k} P_{G_{k}}$, where

$$
c_{4}=c_{5}=c_{10}=1, c_{6}=c_{9}=2
$$

Consider also the line admittance values as

$$
\begin{aligned}
& y_{l m}=-\mathrm{i}, \quad \forall(l, m) \in\{(1,2),(3,4),(4,5),(10,1)\} \\
& y_{l m}=-2 \mathrm{i}, \quad \forall(l, m) \in\{(2,3),(6,7),(9,10)\} \\
& y_{56}=-3 \mathrm{i}, \quad y_{78}=-0.5 \mathrm{i}, y_{89}=-0.7 \mathrm{i}
\end{aligned}
$$

Assume that the load values are equal to

$P_{D_{1}}=-16, P_{D_{2}}=-14, P_{D_{3}}=-18, P_{D_{7}}=P_{D_{8}}=-20$

(the unit is MW). The goal is to solve an OPF problem minimizing the total generation cost subject to the above load constraints and the following network requirements:

- Voltage constraints: $V_{k}^{\text {min }}=0.95$ (per unit) and $V_{k}^{\max }=$ 1.05 for every $k \in \mathcal{N}$, where the base value is 100 MVA.

- Flow constraints: $\Theta_{l m}^{\max }=14^{\circ}$ for every $(l, m) \in \mathcal{L}$.

- Generator constraints: $P_{G_{9}}$ must be less than or equal to 20 MW.

Solving the SDP relaxation for this network yields the optimal cost $\$ 88$, corresponding to the optimal outputs

$P_{G_{4}}=17.8, \quad P_{G_{5}}=32, \quad P_{G_{6}}=P_{G_{9}}=0, \quad P_{G_{10}}=38.2$

The obtained matrix $\mathbf{W}^{\text {opt }}$ has the following eigenvalues:

$$
\begin{aligned}
& 0.0132,0.0146,0.0381,0.0694,0.0896, \\
& 0.2134,0.3167,0.5424,1.4405,7.3939
\end{aligned}
$$

Although the motivation behind using the SDP relaxation was to hopefully obtain a rank-1 solution, the attained matrix $\mathbf{W}^{\text {opt }}$ is full rank with no zero eigenvalues. One may speculate that the relaxation is inexact in this case. To explore this issue, consider the perturbed SDP relaxation for a small nonzero $\epsilon$ (say $\epsilon=10^{-5}$ ). Solving this optimization leads to the optimal cost $\$ 88$, where its solution $\mathbf{W}_{\epsilon}^{\text {opt }}$ has only one nonzero eigenvalue (with value 10.5). The corresponding optimal productions are as follows:

$P_{G_{4}}=24.03, P_{G_{5}}=26.28, P_{G_{6}}=P_{G_{9}}=0, P_{G_{10}}=37.69$

Three conclusions can be made here:

- The SDP relaxation has a hidden rank-1 solution in the sense that a numerical algorithm might produce another solution of this optimization whose rank is high (rank 10 in this case).

- To find the hidden rank-1 solution of the SDP relaxation, the perturbed SDP relaxation can be used.

- The SDP relaxation is exact, but this property may not be easily detected from an optimal primal solution $\mathbf{W}^{\text {opt }}$ or a dual matrix $\mathbf{A}^{\mathrm{opt}}$, unless a small perturbation is applied to the objective function. 


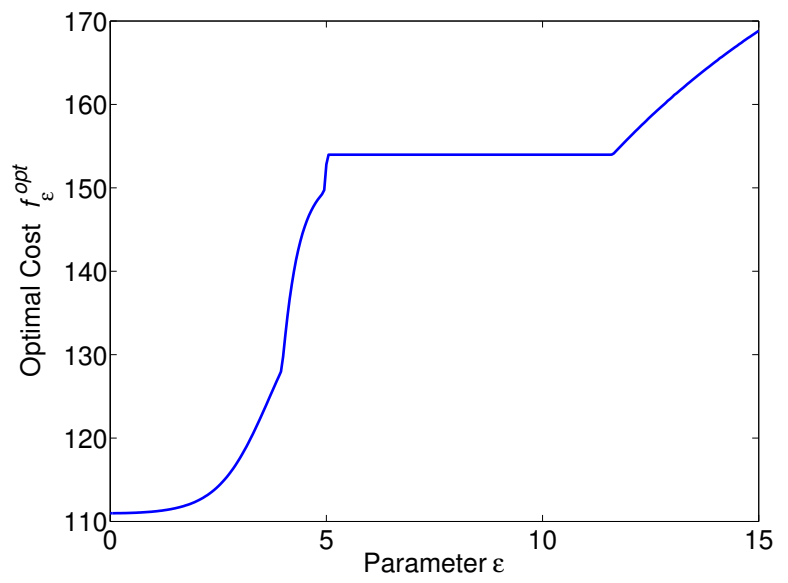

Fig. 2: The optimal cost for the perturbed SDP relaxation as a function of $\epsilon$ for the network studied in Example 2.

Example 2: Consider again the ring network studied in Example 1, but with the following cost coefficients:

$$
c_{4}=10, c_{5}=5, c_{6}=6, c_{9}=9, c_{10}=-3
$$

In this case, the generator at Bus 10 is assumed to have a decreasing cost function. There are various motivations for considering a non-increasing cost function (other than technological constraints) such as the tendency to penetrate as much renewable energy as possible. Solving the SDP relaxation for this network yields the optimal cost $\$ 110.99$, corresponding to a rank-8 matrix $\mathbf{W}^{\text {opt }}$ with the nonzero eigenvalues:

$$
\begin{aligned}
& 0.0003,0.0007,0.0026,0.0035, \\
& 0.0075,0.0104,0.4722,9.5029
\end{aligned}
$$

The associated optimal outputs of the generators at buses $4,5,6,9$ and 10 are:

$$
P_{G_{4}}=P_{G_{6}}=P_{G_{9}}=0, P_{G_{5}}=46.87, P_{G_{10}}=41.13
$$

Since it is not obvious whether or not the SDP relaxation has a hidden rank-1 solution, we solve the perturbed SDP relaxation for different values of $\varepsilon$. Define the optimal cost $f_{\varepsilon}^{\text {opt }}$ as the value of $\sum_{k \in \mathcal{G}} f_{k}\left(P_{G_{k}}\right)$ (and not $\sum_{k \in \mathcal{G}} f_{k}\left(P_{G_{k}}\right)-$ $\left.\varepsilon \sum_{(l, m) \in \mathcal{L}} \operatorname{Re}\left\{W_{l m}\right\}\right)$ at optimality. Figure 2 depicts the optimal cost $f_{\varepsilon}^{\text {opt }}$ for $\varepsilon$ from 0 to 15 . The following observations can be made:

- The obtained numerical solution $\mathbf{W}_{\varepsilon}^{\text {opt }}$ has rank 2 for every $\varepsilon$ in the range $[0,5.03)$.

- The obtained numerical solution $\mathbf{W}_{\varepsilon}^{o p t}$ has rank 1 for every $\varepsilon$ in the range $[5.03,15]$.

- The optimal cost $f_{\varepsilon}^{\text {opt }}$ is fixed at the value $\$ 153.97$ over the relatively wide range $[5.03,11.59]$.

- The optimal cost $f_{\varepsilon}^{\text {opt }}$ for any $\varepsilon$ in the range $[5.03,11.59]$ has the same value as the optimal cost obtained by MATPOWER for the original OPF problem with no perturbation.

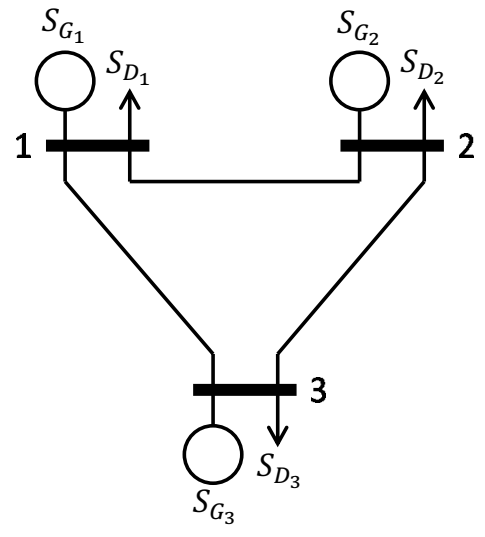

Fig. 3: The three-bus system studied in Example 3.

\begin{tabular}{|l|}
$f_{1}\left(P_{G_{1}}\right) \triangleq 0.11 P_{G_{1}}^{2}+5.0 P_{G_{1}}$ \\
$f_{2}\left(P_{G_{2}}\right) \triangleq 0.085 P_{G_{2}}^{2}+1.2 P_{G_{2}}$ \\
$f_{3}\left(P_{G_{3}}\right) \triangleq 0$ \\
$Z_{23}=0.025+0.750 \mathrm{i}, \quad S_{D_{1}}=110 \mathrm{MW}$ \\
$Z_{31}=0.065+0.620 \mathrm{i}, \quad S_{D_{2}}=110 \mathrm{MW}$ \\
$Z_{12}=0.042+0.900 \mathrm{i}, \quad S_{D_{3}}=95 \mathrm{MW}$ \\
$V_{k}^{\min }=V_{k}^{\max }=1 \quad$ for $\quad k=1,2,3$ \\
$\left(Q_{k}^{\min }, Q_{k}^{\max }\right)=(-\infty, \infty) \quad$ for $\quad k=1,2,3$ \\
$\left(P_{k}^{\min }, P_{k}^{\max }\right)=(-\infty, \infty) \quad$ for $\quad k=1,2$ \\
$P_{3}^{\min }=P_{3}^{\max }=0$
\end{tabular}

TABLE I: Parameters of the three-bus system drawn in Figure 3 with the base value 100 MVA.

This means that whenever the SDP relaxation is inexact, its non-trivial perturbation (for a relatively large value of $\varepsilon$ ) may find a local (yet nearly-global) solution of the original OPF problem.

Example 3: Consider the three-bus system depicted in Figure 3, which has been adopted from [10]. The parameters of this cyclic network are provided in Table I. Assume that lines $(1,2)$ and $(2,3)$ have very high capacities, i.e.,

$$
\begin{aligned}
& \theta_{12}^{\max }=P_{12}^{\max }=S_{12}^{\max }=\Delta V_{12}^{\max }=\infty, \\
& \theta_{23}^{\max }=P_{23}^{\max }=S_{23}^{\max }=\Delta V_{23}^{\max }=\infty,
\end{aligned}
$$

while line $(1,3)$ has a very limited capacity. Since there are at least four ways to limit the flow over this line, we study four problems, each using only one of the capacity constraints given in (3). To this end, given an angle $\alpha$ belonging to the interval $\left[0,90^{\circ}\right]$, consider the following limits for these four problems: 


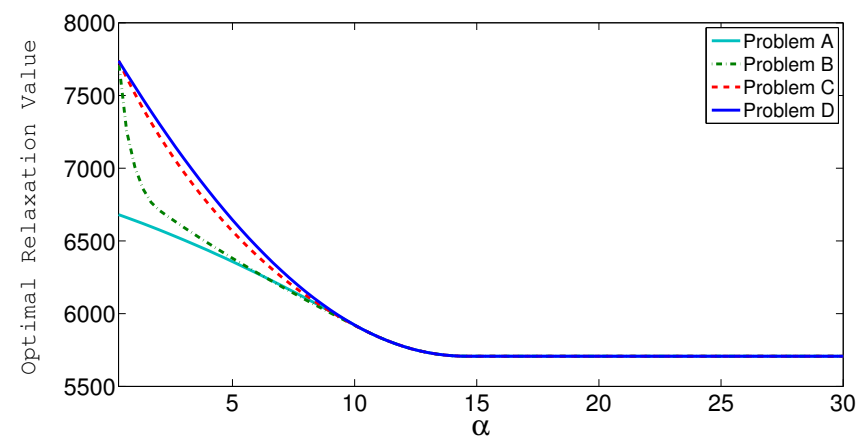

Fig. 4: Optimal objective value of the SDP relaxation for Problems A-D.

$$
\begin{array}{ll}
\text { Problem A : } & \theta_{13}^{\max }=\alpha \\
\text { Problem B : } & P_{13}^{\max }=\operatorname{Re}\left\{\left(1-e^{\alpha \mathrm{i}}\right) y_{13}^{*}\right\} \\
\text { Problem C : } & S_{13}^{\max }=\left|\left(1-e^{\alpha \mathrm{i}}\right) y_{13}^{*}\right| \\
\text { Problem D : } & \Delta V_{13}^{\max }=2(1-\cos (\alpha))
\end{array}
$$

It is straightforward to verify that Problems A, C and D are equivalent due to the fact that they all lead to the same feasible set for the pair $\left(V_{1}, V_{3}\right)$. Moreover, Problem $\mathrm{B}$ is guaranteed to be equivalent to these three problems if $\alpha \leq 10^{\circ}$. However, Problems A-D become very distinct after removing the rank constraint from the OPF problem. To illustrate this property, we solve four SDP problems for the network depicted in Figure 3, corresponding to the equivalent Problems AD. Figure 4 plots the optimal objective value of each of the four SDP relaxations as a function of $\alpha$ over the period $\alpha \in\left[0,30^{\circ}\right]$. Let $f_{\alpha}^{*}$ denote the solution of the original OPF problem. Each of the curves in Figure 4 is theoretically a lower bound on the function $f_{\alpha}^{*}$ in light of removing the nonconvex constraint $\operatorname{rank}\{\mathbf{W}\}=1$. A few observations can be made here:

- The SDP relaxation for Problem D yields a rank-1 solution for all values of $\alpha$. Hence, the curve drawn in Figure 4 associated with Problem D represents the function $f_{\alpha}^{*}$, leading to the true solution of OPF.

- The curves for the SDP relaxations of Problems A-C do not overlap with $f_{\alpha}^{*}$ if $\alpha \in\left(0,7^{\circ}\right)$. Moreover, the gap between these curves and the function $f_{\alpha}^{*}$ is significant for certain values of $\alpha$.

In summary, three types of capacity constraints make the SDP relaxation inexact in general, while the last type of capacity constraint makes the SDP relaxation always exact.

\section{CONCLUSIONS}

We have recently proposed a convex relaxation based on semidefinite programming (SDP) to find the global solution of the optimal power flow (OPF) problem. Although this method has been proven to work for acyclic power networks and IEEE benchmark systems, its limitations for mesh networks have not been fully understood. To address this problem, we provide an upper bound on the rank of the minimum-rank solution of the
SDP relaxation. This number is contingent upon the topology of the power network and is expected to be very small in practice. A heuristic method is then proposed to enforce the SDP relaxation to return a rank-1 solution. To demonstrate the potentials of the SDP relaxation for mesh networks, it is finally shown that this convexification technique always works for weakly-cyclic networks with cycles of size 3. Although the main focus of this paper is placed on OPF, the results can be applied to emerging energy optimizations related to storage and renewable, distributed generation, and demand response for smart grids.

\section{REFERENCES}

[1] J. A. Momoh, M. E. El-Hawary, and R. Adapa, "A review of selected optimal power flow literature to 1993. part i: Nonlinear and quadratic programming approaches," IEEE Transactions on Power Systems, 1999.

[2] I. A. Hiskens and R. J. Davy, "Exploring the power flow solution space boundary," IEEE Transactions on Power Systems, vol. 16, no. 3, pp. 389-395, 2001.

[3] R. Baldick, Applied Optimization: Formulation and Algorithms for Engineering Systems. Cambridge, 2006.

[4] K. S. Pandya and S. K. Joshi, "A survey of optimal power flow methods," Journal of Theoretical and Applied Information Technology, 2008.

[5] J. Lavaei and S. H. Low, "Zero duality gap in optimal power flow problem," IEEE Transactions on Power Systems, vol. 27, no. 1, pp. 92-107, 2012.

[6] J. Lavaei, "Zero duality gap for classical OPF problem convexifies fundamental nonlinear power problems," American Control Conference, 2011.

[7] S. Sojoudi and J. Lavaei, "Physics of power networks makes hard optimization problems easy to solve," IEEE Power \& Energy Society General Meeting, 2012.

[8] B. Zhang and D. Tse, "Geometry of feasible injection region of power networks," 49th Annual Allerton Conference, 2011.

[9] S. Bose, D. F. Gayme, S. Low, and M. K. Chandy, "Optimal power flow over tree networks," Proceedings of the Forth-Ninth Annual Allerton Conference, pp. 1342-1348, 2011.

[10] B. Lesieutre, D. Molzahn, A. Borden, and C. L. DeMarco, "Examining the limits of the application of semidefinite programming to power flow problems," 49th Annual Allerton Conference, 2011.

[11] A. Y. S. Lam, B. Zhang, A. Dominguez-Garcia, and D. Tse, "Optimal distributed voltage regulation in power distribution networks," Submitted for publication, 2012.

[12] Y. Weng, Q. Li, R. Negi, and M. Ilic, "Semidefinite programming for power system state estimation," IEEE Power \& Energy Society General Meeting, 2012.

[13] D. K. Molzahn, B. C. Lesieutre, and C. L. DeMarco, "A sufcient condition for power flow insolvability with applications to voltage stability margins," IEEE Transactions on Power Systems, vol. 28, no. 3, pp. 2592-2601, 2013.

[14] E. Dall'Anese, H. Zhu, and G. Giannakis, "Distributed optimal power flow for smart microgrids," to appear in IEEE Transactions on Smart Grid, 2013.

[15] S. Ghosh, D. A. Iancu, D. Katz-Rogozhnikov, D. T. Phan, and M. S. Squillante, "Power generation management under time-varying power and demand conditions," IEEE Power \& Energy Society General Meeting, 2011.

[16] M. Kraning, E. Chu, J. Lavaei, and S. Boyd, "Dynamic network energy management via proximal message passing," Foundations and Trends in Optimization, vol. 1, no. 2, pp. 1-54, 2013.

[17] D. Gayme and U. Topcu, "Optimal power flow with large-scale storage integration," To appear in IEEE Transactions on Power Systems, 2012.

[18] S. Sojoudi and S. Low, "Optimal charging of plug-in hybrid electric vehicles in smart grids," IEEE Power and Energy Society General Meeting, 2011.

[19] M. Booth, P. Hackney, B. Harris, C. R. Johnson, M. Lay, L. H. Mitchel, S. Narayan, A. Pascoe, K.Steinmetz, B. Sutton, and W. Wang, "On the minimum rank among positive semidefinite matrices with a given graph," SIAM J. on Matrix Analysis and Applications, vol. 30, p. 731740, 2008. 